\title{
On a Generalization of the Stone-Weierstrass Theorem
}

\author{
DIRK HOFMANN \\ Departamento de Matemática, Universidade de Coimbra, 3001-454 Coimbra, Portugal. \\ e-mail:dirk@mat.uc.pt
}

(Received: 20 December 1999; accepted: 10 January 2001)

Abstract. A categorical version of the famous theorem of Stone and Weierstrass is formulated and
studied in detail. Several applications and examples are given.

Mathematics Subject Classifications (2000): 18A05, 18A30, 18A40.

Key words: Stone-Weierstrass theorem, natural duality.

\section{Introduction}

The proof of many duality theorems between concrete categories $(\mathbf{A}, U)$ and $(\mathbf{B}, V)$ over Set can be done by the following steps:

1. Construct a dual adjunction between $(\mathbf{A}, U)$ and $(\mathbf{B}, V)$. Hereby the contravariant functors $G: \mathbf{A} \rightarrow \mathbf{B}$ and $F: \mathbf{B} \rightarrow \mathbf{A}$ of the adjunction are lifts of the hom-functors hom(_, $\tilde{A})$ and hom $\left(\_, \tilde{B}\right)$ represented by objects $\tilde{A} \in \mathrm{Ob} \mathbf{A}$ and $\tilde{B} \in \mathrm{Ob} \mathbf{B}$ with the same carrier $U(\tilde{A})=V(\tilde{B})$.

2. Prove "some" cogenerator property of $\tilde{A}$ and $\tilde{B}$, depending on what lifts we have chosen in the first step.

3. Prove a "Stone-Weierstrass-like" theorem.

It is clear and well studied what is meant by the first two steps. The aim of this paper is to put the latter step in an abstract light. We define and study a condition (Definition 3.4) which exactly states that a "Stone-Weierstrass-like" theorem holds and give several examples and applications. In particular we show how one can extend dualities from the full subcategories of finite objects of given concrete categories $(\mathbf{A}, U)$ and $(\mathbf{B}, V)$ over Set to all objects of $\mathbf{A}$ and $\mathbf{B}$. We apply our results to [3] and prove a general "two-for-one" duality theorem: each strong duality in the sense of [3] gives rise to a new duality by "structure interchange".

In our notation we follow [1]. By a concrete category $(\mathbf{A}, U)$ over $\mathbf{X}$ is meant a category $\mathbf{A}$ together with a faithful functor $U: \mathbf{A} \rightarrow \mathbf{X}$. Since $U$ is injective on hom-sets, we may consider $\operatorname{hom}_{\mathbf{A}}(A, B)$ as a subset of $\operatorname{hom}_{\mathbf{X}}(U(A), U(B))$. It allows us to use the same notation for an A-morphism $f: A \rightarrow B$ and its 
underlying $\mathbf{X}$-morphism $f: U(A) \rightarrow U(B)$. Moreover, we say that a $\mathbf{X}$-morphism $f: U(A) \rightarrow U(B)$ is an A-morphism if it underlies an A-morphism $f: A \rightarrow B$.

\section{Preliminaries}

We recall first some basic facts about concrete dualities, for a detailed discussion see [13]. A dual adjunction

$$
(\mathbf{A}, U) \stackrel{\eta}{\Rightarrow} \underset{F}{\stackrel{G}{\rightleftarrows}} \stackrel{\varepsilon}{\rightleftharpoons}(\mathbf{B}, V)
$$

between concrete categories $(\mathbf{A}, U)$ and $(\mathbf{B}, V)$ over Set is given by contravariant functors $G: \mathbf{A} \rightarrow \mathbf{B}$ and $F: \mathbf{B} \rightarrow \mathbf{A}$ together with natural transformations $\eta: \operatorname{Id}_{\mathbf{A}} \rightarrow F G$ and $\varepsilon: \operatorname{Id}_{\mathbf{B}} \rightarrow G F$ satisfying the equations

$$
G\left(\eta_{A}\right) \circ \varepsilon_{G(A)}=\operatorname{id}_{G(A)} \quad \text { and } \quad F\left(\varepsilon_{B}\right) \circ \eta_{F(B)}=\operatorname{id}_{F(B)}
$$

for each $A \in \mathrm{Ob} \mathbf{A}$ and each $B \in \mathrm{Ob} \mathbf{B}$. We have a dual equivalence - or shorter: a duality - if the units $\eta$ and $\varepsilon$ are natural isomorphisms.

The dual adjunction is induced by $(\tilde{A}, \tilde{B}) \in \mathrm{Ob} \mathbf{A} \times \mathrm{Ob} \mathbf{B}$ if the equations $U(\tilde{A})=V(\tilde{B}), G=\operatorname{hom}\left({ }_{-}, \tilde{A}\right)$ and $F=\operatorname{hom}\left({ }_{-}, \tilde{B}\right)$ hold and the units $\eta$ and $\varepsilon$ are given by

$$
\eta_{A}: A \rightarrow F G(A), a \mapsto \mathrm{ev}_{A, a} \quad \text { and } \quad \varepsilon_{B}: B \rightarrow G F(B), b \mapsto \mathrm{ev}_{B, b}
$$

for each $A \in \mathrm{Ob} \mathbf{A}$ and $B \in \mathrm{Ob} \mathbf{B}$. Hereby $\mathrm{ev}_{A, a}$ denotes the evaluation map $\operatorname{ev}_{A, a}: \operatorname{hom}(A, \tilde{A}) \rightarrow U(\tilde{A})=V(\tilde{B}), h \mapsto h(a)$ for each $A \in \mathrm{Ob} \mathbf{A}$ and each $a \in$ $U(A)$ and, symmetrically, $\operatorname{ev}_{B, b}$ denotes the evaluation map $\operatorname{ev}_{B, b}: \operatorname{hom}(B, \tilde{B}) \rightarrow$ $U(\tilde{A}), h \mapsto h(b)$ for each $B \in \mathrm{Ob} \mathbf{B}$ and each $b \in V(B)$. If the functors $U: \mathbf{A} \rightarrow$ Set and $V: \mathbf{B} \rightarrow$ Set are representable and uniquely transportable, each dual adjunction between $(\mathbf{A}, U)$ and $(\mathbf{B}, V)$ essentially has this structure. The forgetful functor to Set of many familiar categories has this property, hence it is no restriction at all to assume that all concrete categories over Set in this article are of this kind.

Assume that a dual adjunction

$$
(\mathbf{A}, U) \stackrel{\eta}{\Rightarrow} \underset{F}{\stackrel{G}{\rightleftarrows}} \stackrel{\varepsilon}{\rightleftarrows}(\mathbf{B}, V)
$$

induced by $(\tilde{A}, \tilde{B})$ is given. For each $A \in \mathrm{Ob} \mathbf{A}$ we can define a hom $(\tilde{A}, \tilde{A})$-action

$$
\tau_{A}: \operatorname{hom}(\tilde{A}, \tilde{A}) \times \operatorname{hom}(A, \tilde{A}) \rightarrow \operatorname{hom}(A, \tilde{A}),(h, f) \mapsto h \circ f
$$

on $\operatorname{hom}(A, \tilde{A})$. We consider now the question when B-morphisms preserve this additional structure.

LEMMA 1.1. The following are equivalent. 
(1) $\eta_{\tilde{A}}$ is an isomorphism.

(2) For each $A \in \mathrm{Ob} \mathbf{A}$, all A-morphisms $h: \tilde{A} \rightarrow \tilde{A}$ and $f: A \rightarrow \tilde{A}$ and each B-morphism $\varphi: G(A) \rightarrow \tilde{B}$, the equation $\varphi(h \circ f)=h(\varphi(f))$ holds.

Proof. (1) $\Rightarrow$ (2) We consider the B-morphism

$$
G(\tilde{A}) \stackrel{G(f)}{\rightarrow} G(A) \stackrel{\varphi}{\rightarrow} \tilde{B}
$$

Since $\eta_{\tilde{A}}$ is an isomorphism there exists an element $a \in U(\tilde{A})$ such that $\varphi \circ G(f)=$ $\mathrm{ev}_{\tilde{A}, a}$. We have

$$
a=\operatorname{ev}_{\tilde{A}, a}\left(\operatorname{id}_{\tilde{A}}\right)=(\varphi \circ G(f))\left(\operatorname{id}_{\tilde{A}}\right)=\varphi(f)
$$

and therefore

$$
\varphi(h \circ f)=(\varphi \circ G(f))(h)=\mathrm{ev}_{\tilde{A}, a}(h)=h(a)=h(\varphi(f)) .
$$

(2) $\Rightarrow$ (1) Let $\varphi: G(\tilde{A}) \rightarrow \tilde{B}$ be a B-morphism and $a=\varphi\left(\operatorname{id}_{\tilde{A}}\right)$. For each $f \in \operatorname{hom}(\tilde{A}, \tilde{A})$ we have

$$
\varphi(f)=\varphi\left(f \circ \operatorname{id}_{\tilde{A}}\right)=f\left(\varphi\left(\operatorname{id}_{\tilde{A}}\right)\right)=f(a),
$$

hence $\varphi=\operatorname{ev}_{\tilde{A}, a}$.

COROLLARY 1.2. Assume that $\eta_{\tilde{A}}$ is an isomorphism. For all $\mathbf{A}$-objects $A$ and $B$, all A-morphisms $h: \tilde{A} \rightarrow \tilde{A}$ and $f: A \rightarrow \tilde{A}$ and each $\mathbf{B}$-morphism $\psi:$ $G(A) \rightarrow G(B)$, the equation $\psi(h \circ f)=h \circ \psi(f)$ holds.

So far we have studied the structure of a given dual adjunction. But how can we construct a dual adjunction between given concrete categories $(\mathbf{A}, U)$ and $(\mathbf{B}, V)$ over Set? Certainly we have to find objects $\tilde{A} \in \mathrm{Ob} \mathbf{A}$ and $\tilde{B} \in \mathrm{Ob} \mathbf{B}$ with the same carrier $U(\tilde{A})=V(\tilde{B})$ such that

(1) for each $A \in \mathrm{Ob} \mathbf{A}$, the $V$-structured source $\left(\operatorname{ev}_{A, a}: \operatorname{hom}(A, \tilde{A}) \rightarrow V(\tilde{B})\right)_{a \in U(A)}$ admits a $V$-lifting $\left(\mathrm{ev}_{A, a}: G(A) \rightarrow \tilde{B}\right)_{a \in U(A)}$ such that, for each $f: A \rightarrow A^{\prime}$ in $\mathbf{A}$, the map hom $(f, \tilde{A})$ underlies a $\mathbf{B}$-morphism $G(f)$,

(2) for each $B \in \mathrm{Ob} \mathbf{B}$, the $U$-structured source $\left(\operatorname{ev}_{B, b}: \operatorname{hom}(B, \tilde{B}) \rightarrow U(\tilde{A})\right)_{b \in V(B)}$ admits a $U$-lifting $\left(\operatorname{ev}_{B, b}: F(B) \rightarrow \tilde{A}\right)_{b \in V(B)}$ such that, for each $g: B \rightarrow B^{\prime}$ in $\mathbf{B}$, the map hom $(g, \tilde{B})$ underlies an $\mathbf{A}$-morphism $F(g)$,

(3) for each $A \in \mathrm{Ob} \mathbf{A}$, the map

$$
\eta_{A}: U(A) \rightarrow U F G(A)=\operatorname{hom}(G(A), \tilde{B}), a \mapsto \mathrm{ev}_{A, a}
$$

is actually an A-morphism $\eta_{A}: A \rightarrow F G(A)$ and

(4) for each $B \in \mathrm{Ob} \mathbf{B}$, the map

$$
\varepsilon_{A}: V(B) \rightarrow V G F(B)=\operatorname{hom}(F(B), \tilde{A}), b \mapsto \operatorname{ev}_{B, b}
$$

is actually a $\mathbf{B}$-morphism $\varepsilon_{B}: B \rightarrow G F(B)$. 
If the sources $\left(\operatorname{ev}_{A, a}: \operatorname{hom}(A, \tilde{A}) \rightarrow V(\tilde{B})\right)_{a \in U(A)}$ and $\operatorname{(ev}_{B, b}: \operatorname{hom}(B, \tilde{B}) \rightarrow$ $U(\tilde{A}))_{b \in V(B)}$ admit a $V$-resp. $U$-initial lifting, all other conditions are automatically fulfilled. Therefore we consider the following conditions.

(Init A) For each $A \in \mathrm{Ob} \mathbf{A}$, the $V$-structured source $\left(\operatorname{ev}_{A, a}: \operatorname{hom}(A, \tilde{A}) \rightarrow\right.$ $V(\tilde{B}))_{a \in U(A)}$ admits a $V$-initial lifting $\left(\mathrm{ev}_{A, a}: G(A) \rightarrow \tilde{B}\right)_{a \in U(A)}$.

(Init B) For each $B \in \mathrm{Ob} \mathbf{B}$, the $U$-structured source $\left(\operatorname{ev}_{B, b}: \operatorname{hom}(B, \tilde{B}) \rightarrow\right.$ $U(\tilde{A}))_{b \in V(B)}$ admits a $U$-initial lifting $\left(\operatorname{ev}_{B, a}: F(B) \rightarrow \tilde{A}\right)_{b \in V(B)}$.

A dual adjunction induced by $(\tilde{A}, \tilde{B})$ is called a natural dual adjunction if the sources $\left(\operatorname{ev}_{A, a}: G(A) \rightarrow \tilde{B}\right)_{a \in U(A)}$ and $\left(\operatorname{ev}_{B, a}: F(B) \rightarrow \tilde{A}\right)_{b \in V(B)}$ are initial with respect to $V$ and $U$ respectively. For a natural dual adjunction induced by $(\tilde{A}, \tilde{B})$ we have that, for each $A \in \mathrm{Ob} \mathbf{A}, \eta_{A}$ is an embedding if and only if the source $\operatorname{hom}(A, \tilde{A})$ is point separating and $U$-initial. This suggests the following definition.

DEFINITION 1.3. Let $(\mathbf{A}, U)$ be a concrete category over Set and let $\tilde{A} \in$ $\mathrm{Ob} \mathbf{A}$. $\tilde{A}$ is called initial cogenerator of $(\mathbf{A}, U)$ if, for each $A \in \mathrm{Ob} \mathbf{A}$, the source $\operatorname{hom}(A, \tilde{A})$ is point separating and $U$-initial.

Of course, if the forgetful functors $U$ and $V$ are mono-topological the conditions (Init A) and (Init B) are fulfilled. But what about the algebraic case? Recall that a signature is a pair $\Sigma=\left(\Sigma_{\mathrm{op}}, \delta\right)$ consisting of a class $\Sigma_{\mathrm{op}}$ of operation symbols and an arity function $\delta: \Sigma_{\mathrm{op}} \rightarrow$ Card. $\Sigma=\left(\Sigma_{\mathrm{op}}, \delta\right)$ is called finitary if $\Sigma_{\mathrm{op}}$ is a set and, for each $\theta \in \Sigma_{\mathrm{op}}, \delta(\theta) \in \mathbb{N}$. A $\Sigma$-algebra is a pair $B=\left(|B|, \delta^{B}\right)$ consisting of a set $|B|$ and a map $\delta^{B}$ assigning to each $\theta \in \Sigma_{\text {op }}$ an operation $\delta^{B}(\theta)=\theta_{B}:|B|^{\sigma(\theta)} \rightarrow|B|$ of arity $\sigma(\theta)$ on $|B|$. Let $B_{1}=\left(\left|B_{1}\right|, \delta^{B_{1}}\right)$ and $B_{2}=\left(\mid B_{2}, \delta^{B_{2}}\right)$ be $\Sigma$-algebras. A map $f:\left|B_{1}\right| \rightarrow\left|B_{2}\right|$ is a $\Sigma$-homomorphism $f: B_{1} \rightarrow B_{2}$ provided that $\theta_{B_{2}} \circ f^{\sigma(\theta)}=f \circ \theta_{B_{1}}$ for each $\theta \in \Sigma_{\text {op. }}$. Alg $\Sigma$ denotes the category of all $\Sigma$-algebras and all $\Sigma$-homomorphisms.

Let $B=\left(|B|, \delta^{B}\right)$ be a $\Sigma$-algebra and let $X$ be a set. $\mathrm{Cl}_{X}(B)$ denotes the $\Sigma$ algebra of all $X$-ary term functions on $B$. It is the smallest subalgebra of $B^{\left(|B|^{X}\right)}$ containing all projections $\pi_{x}:|B|^{X} \rightarrow|B|$. The algebra $\mathrm{Cl}_{X}(B)$ is called the clone algebra of $X$-ary term functions on $B$.

DEFINITION 1.4. Let $(\mathbf{A}, U)$ be a concrete category over Set and $\tilde{A} \in \mathrm{Ob} \mathbf{A}$. $(\mathbf{A}, U)$ is called concretely $\tilde{A}$-complete if all powers of $\tilde{A}$ exist in $\mathbf{A}$ and all equalizers of pairs of morphisms between powers of $\tilde{A}$ exist in $\mathbf{A}$ and $U$ preserve those limits.

LEMMA 1.5. Let $(\mathbf{A}, U)$ be a concrete category over $\mathbf{S e t}$, let $\tilde{A}$ be an $\mathbf{A}$-object with arbitrary concrete powers and let $\mathbf{B}=\{B \in \operatorname{Ob} \operatorname{Alg} \Sigma \mid \operatorname{hom}(B, \tilde{B})$ is a mono-source $\}$ be the quasi-variety cogenerated by a $\Sigma$-algebra $\tilde{B}$ for a given signature $\Sigma$. Further $V: \mathbf{B} \rightarrow$ Set denotes the canonical forgetful functor and we assume that $U(\tilde{A})=V(\tilde{B})$. 
(1) The following are equivalent.

(a) (Init A) holds.

(b) For each set $X, V\left(\mathrm{Cl}_{X}(\tilde{B})\right) \subset U\left(\operatorname{hom}\left(\tilde{A}^{X}, \tilde{A}\right)\right)$.

(2) Assume, in addition, that $(\mathbf{A}, U)$ is concretely $\tilde{A}$-complete. For each $B \in$ $\operatorname{Ob} \operatorname{Alg} \Sigma$, the canonical inclusion $\operatorname{hom}(B, \tilde{B}) \subset U(\tilde{A})^{V(B)}$ is the equalizer of a pair of $\mathbf{A}$-morphisms between powers of $\tilde{A}$ and therefore underlies an $\mathbf{A}$-object. In particular, (Init A) implies (Init B).

We now consider our motivating example.

\section{Gelfand-Duality}

Let $\mathbf{A}=\mathbf{C o m p}_{2}$ be the category of compact Hausdorff spaces and continuous maps. $U: \mathbf{A} \rightarrow$ Set denotes the usual forgetful functor. $\mathbf{B}=C^{*}$-Alg is the category with all commutative $C^{*}$-algebras with identity as objects and identity- and _- ${ }^{*}$ preserving $\mathbb{C}$-algebra homomorphisms as morphisms. $V=\bigcirc: \mathbf{B} \rightarrow$ Set denotes the unit-ball functor. Note that in both categories the class of all embeddings coincides with the class of all monomorphisms. Let $\tilde{A}=\mathbb{D}$ be the unit disk and $\tilde{B}=\mathbb{C}$ the $C^{*}$-algebra of complex numbers. Obviously we have $U(\mathbb{D})=\bigcirc(\mathbb{C})$.

The Gelfand-duality theorem states that $(\mathbb{D}, \mathbb{C})$ induces a dual equivalence between the categories $\mathbf{C o m p}_{2}$ and $C^{*}$-Alg.

For each compact Hausdorff space $A$, the set $C(A)$ of all complex valued continuous functions endowed with pointwise defined operations and the supremum norm $\|f\|_{0}=\sup \{|f(x)| \mid x \in U(A)\}$ is a $C^{*}$-algebra, the source $\left(\mathrm{ev}_{A, a}: C(A)\right.$ $\rightarrow \mathbb{C})_{a \in U(A)}$ being a $\bigcirc$-initial lift of the source $\left(\operatorname{ev}_{A, a}: \operatorname{hom}(A, \mathbb{D}) \rightarrow \mathbb{D}=\right.$ $\bigcirc(\mathbb{C}))_{a \in U(A)}$. Hence the condition (Init $\mathbf{A}$ ) is fulfilled.

For each $C^{*}$-algebra $B$, we can define the initial topology on $\operatorname{hom}(B, \mathbb{C})$ with respect to the $U$-structured source $\left(\operatorname{ev}_{B, b}: \operatorname{hom}(B, \mathbb{C}) \rightarrow U(\mathbb{D})\right)_{b \in \mathrm{O}(B)}$. This topology turns out to be compact and Hausdorff (see Lemma 1.5), (Init $\mathbf{B}$ ) is also fulfilled.

Therefore we get a natural dual adjunction

$$
\left(\mathrm{Comp}_{2}, U\right) \stackrel{\eta}{\Rightarrow} \underset{S}{\stackrel{C}{\gtrless}} \stackrel{\varepsilon}{\rightleftarrows}\left(C^{*}-\mathbf{A l g}, \bigcirc\right)
$$

induced by $(\mathbb{D}, \mathbb{C})$.

From the Urysohn lemma we know that $\tilde{A}=\mathbb{D}$ is a cogenerator of $\mathbf{A}$. The corresponding result about the $C^{*}$-algebra $\mathbb{C}$ is a consequence of the following well-known fact (see [5]).

PROPOSITION 2.1. For each $C^{*}$-algebra $B$ and each element $x \in B$,

$$
\|x\|=\sup \{|\varphi(x)| \mid \varphi \in \operatorname{hom}(B, \mathbb{C})\} .
$$


Hence each B-morphism has norm not greater than 1 and $\tilde{B}=\mathbb{C}$ is a cogenerator of $\mathbf{B}$. We conclude that the units $\eta$ and $\varepsilon$ are pointwise monomorphisms.

The Stone-Weierstrass theorem $([11,12,15])$ implies that $\varepsilon$ is actually a natural isomorphism.

THEOREM 2.2 (Stone-Weierstrass). Let A be a compact Hausdorff space and let $M \subset C(A)$ be a $C^{*}$-subalgebra of $C(A)$ such that the source $(f: A \rightarrow \mathbb{D})_{f \in \mathrm{O}(M)}$ separates the points of $A$. Then $M=C(A)$.

For each $C^{*}$-algebra $B$, the requirements of this theorem are fulfilled with $A=$ $S(B)$ and $M$ the image of $\varepsilon_{B}$. Therefore $\varepsilon_{B}$ is surjective and hence an isomorphism. To prove that $\eta$ is a natural isomorphism we can proceed in a similar way.

PROPOSITION 2.3. Let $B$ be a $C^{*}$-algebra and let $M \subset S(B)$ be a closed subspace of $S(B)$ such that the source $(f: B \rightarrow \mathbb{C})_{f \in U(M)}$ separates the points of $B$. Then $M=S(B)$.

Proof. From the Stone-Weierstrass theorem we know that, for each $C^{*}$-algebra $B, \varepsilon_{B}$ and hence $\eta_{F(B)}$ is an isomorphism. It follows that $\eta_{\mathbb{D}}$ is an isomorphism and we can apply Lemma 1.1 .

Let $B$ be a $C^{*}$-algebra. We define the Zariski-topology on $\operatorname{hom}(B, \mathbb{C})$ by

$$
\psi \in \operatorname{cl}_{\mathcal{Z}} \Phi \Leftrightarrow \mathcal{T}_{\mathcal{Z}}(\psi, \Phi) \quad \text { for all } \psi \in \operatorname{hom}(B, \mathbb{C}) \text { and } \Phi \subset \operatorname{hom}(B, \mathbb{C}) .
$$

Hereby the formula $\mathcal{T}_{\mathcal{Z}}(\psi, \Phi)$ is defined as follows:

$$
\mathcal{T}_{\mathcal{Z}}(\psi, \Phi) \equiv \forall x \in \bigcirc(B)((\forall \varphi \in \Phi \varphi(x)=0) \rightarrow \psi(x)=0) .
$$

Obviously, in this topology the point separating subsets are precisely the dense subsets. We are going to show that this topology coincides with the initial topology with respect to the source of all evaluation maps. To do this, we consider the formula

$$
\mathcal{T}_{\ell}(\psi, \Phi) \equiv \forall x \in \bigcirc(B) \psi(x) \in \overline{\{\varphi(x) \mid \varphi \in \Phi\}} .
$$

Hereby $\bar{A}$ denotes the closure of a subset $A \subset \mathbb{D}$ in $\mathbb{D}$. We claim that, for each $\psi \in \operatorname{hom}(B, \mathbb{C})$ and each $\Phi \subset \operatorname{hom}(B, \mathbb{C}), \mathcal{T}_{\mathcal{Z}}(\psi, \Phi)$ holds if and only if $\mathcal{T}_{l}(\psi, \Phi)$ holds.

Let $\psi \in \operatorname{hom}(B, \mathbb{C})$ and $\Phi \subset \operatorname{hom}(B, \mathbb{C})$. If $\Phi=\emptyset$, both formulas are false. Hence we can assume that $\Phi \neq \emptyset$. Moreover, without loss of generality we may assume that $B=C(A)$ for a compact Hausdorff space $A$.

Assume first that $\mathcal{T}_{l}(\psi, \Phi)$ holds and let $x \in \bigcirc(B)$ such that $\varphi(x)=0$ for all $\varphi \in \Phi$. We have $\psi(x) \in \overline{\{0\}}=\{0\}$.

Assume now that $\mathcal{T}_{\ell}(\psi, \Phi)$ is false. There exists an element $x: A \rightarrow \mathbb{D} \in$ $\bigcirc(B)$ such that $\psi(x) \notin \overline{\{\varphi(x) \mid \varphi \in \Phi\}}$ holds. Since $\mathbb{D}$ is totally regular, there exists a continuous map $h: \mathbb{D} \rightarrow \mathbb{D}$ such that $h(\psi(x)) \neq 0$ and $h[\{\varphi(x) \mid \varphi \in \Phi\}]$ 
$=\{h(\varphi(x)) \mid \varphi \in \Phi\}=\{0\}$. By Lemma 1.1 we have $\psi(h \circ x) \neq 0$ and $\varphi(h \circ x)=0$ for all $\varphi \in \Phi$. Hence $\mathcal{T}_{\mathcal{Z}}(\psi, \Phi)$ is false as well.

\section{The Stone-Weierstrass Condition}

In the latter section we have proved the Gelfand-duality theorem by two "StoneWeierstrass theorems". It turns out that the proof of many duality theorems can be done in the same way (see Examples 3.5). We take this as a motivation to formulate and study in this section precisely what is meant by a "Stone-Weierstrass-like" theorem.

Let us first collect some facts about factorization systems. For more details see [1]. Let $\mathbf{C}$ be a complete category and let $\mathbb{M}$ be a class of $\mathbf{C}$-morphisms satisfying the following conditions:

1. $\operatorname{Section}(\mathbf{C}) \subset \mathbb{M} \subset \operatorname{Mono}(\mathbf{C})$,

2. $\mathbb{M}$ is closed under composition, stable under pullbacks and

3. for each family $\left(m_{i}: A_{i} \rightarrow A\right)_{i \in I}$ of $\mathbb{M}$-morphisms, there exist an intersection $d: D \rightarrow A$ and $d \in \mathbb{M}$.

In most cases we will choose $\mathbb{M}$ as the class of all embeddings of a concretely complete concrete category $(\mathbf{C}, W)$ over Set. But also $\mathbb{M}=\operatorname{RegMono}(\mathbf{C})$ can be a reasonable choice.

Any such class $\mathbb{M}$ of $\mathbf{C}$-morphisms satisfying $(*)$ is part of a factorization structure (M-ExtrEpiSink, $\mathbb{M}$ ) for sinks and of a factorization structure (M-ExtrEpi, $\mathbb{M}$ ) for morphisms in $\mathbf{C}$. Moreover, we define the following class of small sources of $\mathbf{C}$ :

$$
\mathcal{M}=\left\{\left(f_{i}: C \rightarrow C_{i}\right)_{i \in I} \mid I \text { is a set and }\left\langle f_{i}\right\rangle_{i \in I} \in \mathbb{M}\right\} .
$$

Obviously, $\mathcal{M}$ is closed under composition, each limit source belongs to $\mathcal{M}$ and a small source belongs to $\mathcal{M}$ if and only if it contains a $\mathcal{M}$-source.

DEFINITION 3.1. Let $\tilde{C}$ be a $\mathbf{C}$-object. $\tilde{C}$ is called an $\mathbb{M}$-cogenerator of $\mathbf{C}$ if, for each $C \in \mathrm{Ob} \mathbf{C}$, the source $\operatorname{hom}(C, \tilde{C})$ belongs to $\mathcal{M}$.

In the sequel we will often make use of the following fact. Assume that $\tilde{C}$ is a regular cogenerator of $\mathbf{C}$. It follows that, for each $C \in \mathrm{Ob} \mathbf{C}$, there exists an equalizer diagram

$$
C \stackrel{e}{\longrightarrow} \tilde{C}^{X} \underset{g}{\stackrel{f}{\longrightarrow}} \tilde{C}^{Y}
$$

with sets $X$ and $Y$ and $\mathbf{C}$-morphisms $f$ and $g$. Hence any right adjoint, full and faithful functor $F: \mathbf{B} \rightarrow \mathbf{C}$ is an equivalence provided that $\tilde{C}$ is, up to isomorphism, contained in the image of $F$. 
ASSUMPTION 3.2. Let $(\mathbf{A}, U)$ and $(\mathbf{B}, V)$ be concretely complete concrete categories over Set and let $\tilde{A} \in \mathrm{Ob} \mathbf{A}$ and $\tilde{B} \in \mathrm{Ob} \mathbf{B}$ be objects with the same underlying set $U(\tilde{A})=V(\tilde{B})$. Furthermore there are given classes $\mathbb{M}_{\mathbf{A}}$ and $\mathbb{M}_{\mathbf{B}}$ of A-resp. B-morphisms satisfying $(*)$. We assume that $(\tilde{A}, \tilde{B})$ induces a dual adjunction

$$
(\mathbf{A}, U) \stackrel{\eta}{\Rightarrow} \underset{F}{\stackrel{G}{\rightleftarrows}} \stackrel{\varepsilon}{\rightleftharpoons}(\mathbf{B}, V)
$$

such that, for each $A \in \mathrm{Ob} \mathbf{A}$ and each $B \in \mathrm{Ob} \mathbf{B}$, the sources $\left(\mathrm{ev}_{A, a}: G(A) \rightarrow\right.$ $\tilde{B})_{a \in U(A)}$ and $\left(\operatorname{ev}_{B, b}: F(B) \rightarrow \tilde{A}\right)_{b \in V(B)}$ belong to $\mathcal{M}_{\mathbf{A}}$ resp. $\mathcal{M}_{\mathbf{B}} . \tilde{A}$ is a $\mathbb{M}_{\mathbf{A}^{-}}$ cogenerator of $\mathbf{A}$ and $\tilde{B}$ is a $\mathbb{M}_{\mathbf{B}}$-cogenerator of $\mathbf{B}$.

The situation described above is our basic situation. Throughout this paper we will always assume that it is given. We have the following obvious facts.

PROPOSITION 3.3. (1) The following are equivalent.

(a) $G\left(\mathcal{M}_{\mathbf{A}}\right) \subset \mathbb{M}_{\mathbf{B}}$-ExtrEpiSink.

(b) $F\left(\mathcal{M}_{\mathbf{B}}\right) \subset \mathbb{M}_{\mathbf{A}}-$ ExtrEpiSink.

(2) The following are equivalent.

(a) $G\left(\mathbb{M}_{\mathbf{A}}\right.$-ExtrEpiSink) $\subset \mathcal{M}_{\mathbf{B}}$.

(b) $F\left(\mathbb{M}_{\mathbf{B}}-\right.$ ExtrEpiSink $) \subset \mathcal{M}_{\mathbf{A}}$.

We come now to the central definition of this paper. We will formulate it only with respect to $G$, by symmetry, there is a corresponding formulation with respect to $F$.

DEFINITION 3.4. G satisfies the Stone-Weierstrass Condition provided that the following holds:

(SW) For each A-object $A$, every $\mathbb{M}_{\mathbf{B}}$-morphism $m: M \rightarrow G(A)$ is an isomorphism provided that $(m(f))_{f \in V(M)} \in \mathcal{M}_{\mathbf{A}}$.

EXAMPLES 3.5. All categories in the sequel are equipped with a canonical forgetful functor to Set. To simplify our notation we will not denote these functors. In all examples we will choose $\mathbb{M}_{\mathbf{A}}$ and $\mathbb{M}_{\mathbf{B}}$ as the class of all embeddings. Lemma 1.5 implies in all examples that the conditions (Init A) and (Init B) are fulfilled.

(1) (Stone-duality, [10]) Let $\mathbf{A}=$ Stone be the category of zero-dimensional compact Hausdorff spaces and let $\mathbf{B}=\mathbf{B o o l}$ be the category of Boolean algebras. $\tilde{A}$ denotes the discrete two-element spaces $(\{0,1\}, \mathcal{P}(\{0,1\}))$ and $\mathbf{B}$ the 2-chain. Note that in both categories the class of all embeddings coincides with the class of all monomorphisms. It is clear by definition that $\tilde{A}$ is a cogenerator of Stone. 
The Prime Ideal Theorem implies that $\tilde{B}$ is a cogenerator of Bool. $(\tilde{A}, \tilde{B})$ induces a natural dual adjunction between Stone and Bool. We are going to show that the contravariant functor $G$ : Stone $\rightarrow$ Bool satisfies (SW).

Let $A \in \mathrm{Ob} \mathbf{A}$ be an $\mathbf{A}$-object and let $M \hookrightarrow G(A)$ be a subalgebra of $G(A)$ such that the source $(h: A \rightarrow \tilde{A})_{h \in M}$ separates the points of $A$. Let $f: A \rightarrow \tilde{A}$ be any continuous map. We have to show that $f \in M$. Since $M$ contains the constant maps, we may assume that the sets $A_{0}=f^{-1}[\{0\}]$ and $A_{1}=f^{-1}[\{1\}]$ are non-empty. For each $x \in A_{0}$ and each $y \in A_{1}$, there exists $h_{x, y} \in M$ such that $h_{x, y}(x)=0$ and $h_{x, y}(y)=1$. Hereby we use the fact that $M$ is point separating and that with each $k \in M$ also the complement $\bar{k}$ is contained in $M$. Since $A$ is compact, for each $y \in A_{1}$, there exist finitely many elements $x_{1}(y), \ldots, x_{n_{y}}(y) \in A_{0}$ with the property that, for each $z \in A_{0}$, there is an index $i \in\left\{1, \ldots, n_{y}\right\}$ such that $h_{x_{i}(y), y}(z)=0$. We put

$$
h_{y}=\bigwedge_{i=1}^{n_{y}} h_{x_{i}(y), y} \in M .
$$

For each $y \in A_{1}$ we have $h_{y}(y)=1$ and $h_{y}(x)=0$ for each $x \in A_{0}$. Since $A$ is compact there exist finitely many $y_{1}, \ldots, y_{n}$ such that, for each $y \in A_{1}$, there is an index $i \in\{1, \ldots, n\}$ such that $h_{y_{i}}(y)=1$. We put

$$
h=\bigvee_{i=1}^{n} h_{y_{i}} \in M .
$$

Obviously we have $h=f$.

Hence the contravariant functor $F:$ Bool $\rightarrow$ Stone is full and faithful. One can now prove similarly to Proposition 2.3 that $F$ satisfies (SW). Therefore we have a dual equivalence between Stone and Bool. However, the following argument is shorter. Let $B_{0} \in \mathrm{Ob}$ Bool be any object representing the canonical forgetful functor from Bool to Set. $\tilde{A}$ is isomorphic to $F\left(B_{0}\right)$ and, moreover, a regular cogenerator of Stone. We conclude that $F$ is an equivalence functor.

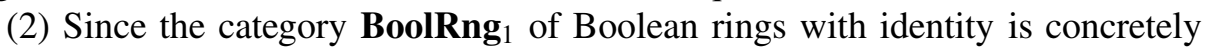
isomorphic to Bool, we may consider $\mathbf{B}=\mathbf{B o o l R n g}_{1}$ and $\tilde{B}=\mathbb{Z} / 2 \mathbb{Z}$. $(\tilde{A}, \tilde{B})$ in-

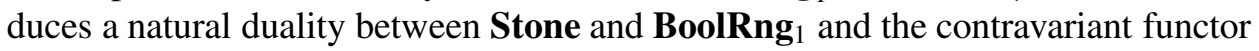
$G:$ Stone $\rightarrow$ BoolRng $_{1}$ satisfies (SW).

(3) (Priestley-duality, $[8,9])$ StonePos denotes the category of ordered Stonespaces and continuous, order preserving maps and $\tilde{A}$ the 2-chain provided with the discrete topology. From [14] we know that $\tilde{A}$ is not an initial cogenerator of StonePos. A = Priest denotes the full subcategory of StonePos initially cogenerated by $\tilde{A}$, which means that we have

Priest $=\{A \in \mathrm{ObStonePos} \mid \operatorname{hom}(A, \tilde{A})$ is point separating and initial $\}$.

The objects of Priest are called Priestley spaces. Further we put $\mathbf{B}=$ DLat $_{0,1}$, the category of bounded distributive lattices and homomorphisms and $\tilde{B}$ is the 
2-chain. It is well-known (again the Prime Ideal Theorem) that $\tilde{B}$ is a cogenerator of DLat $_{0,1}$. $(\tilde{A}, \tilde{B})$ induces a natural dual adjunction between Priest and DLat $t_{0,1}$. We will show that $G:$ Priest $\rightarrow$ DLat $_{0,1}$ satisfies (SW). The proof is similar to the first example, in particular it becomes clear how the additional order-structure on one side compensates the lack of the negation operator on the other side.

Let $A$ be a Priestley space and let $M \hookrightarrow G(A)$ be a sublattice of $G(A)$ such that the source $(h)_{h \in M}$ is point separating and initial. Note that a source $\left(f_{i}: A \rightarrow\right.$ $\left.A_{i}\right)_{i \in I}$ is initial in Priest if and only if

$$
x \leqslant y \Leftrightarrow \forall i \in I f_{i}(x) \leqslant f_{i}(y)
$$

for all $x, y \in A$. Let $f: A \rightarrow \tilde{A}$ be a non-constant Priest-morphism, we define $A_{0}=f^{-1}[\{0\}] \neq \emptyset$ and $A_{1}=f^{-1}[\{1\}] \neq \emptyset$. For each $(x, y) \in A_{0} \times A_{1}$ holds $x \ngtr y$, hence there exists $h_{x, y} \in M$ such that $h_{x, y}(x)=0$ and $h_{x, y}(y)=1$. As in the first example one can now prove that $f \in M$.

We will see later on (Example 4.10) that $\tilde{A}$ is a regular cogenerator of Priest, hence we can conclude that Priest is dually equivalent to DLat $\mathbf{D}_{0,1}$.

(4) We now put $\mathbf{A}=$ pStone, $\mathbf{B}=$ BoolRng, $\tilde{A}=(\{0,1\}, 0)$ and $\tilde{B}=\mathbb{Z} / 2 \mathbb{Z}$. Hereby pStone denotes the category of pointed Stone-spaces and point-preserving continuous maps and BoolRng the category of Boolean rings (not necessary with

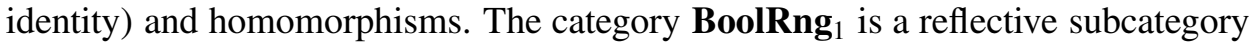
of BoolRng, the reflection map being given by

$$
B \hookrightarrow B \cup\{1+x \mid x \in B\} .
$$

From that we conclude that $\tilde{B}$ is an injective cogenerator of BoolRng. $\tilde{A}$ is obviously a cogenerator of pStone. Note that in both categories the class of all monomorphisms coincides with the class of all embeddings. $(\tilde{A}, \tilde{B})$ induces a natural dual adjunction between pStone and BoolRng. We will show that the contravariant functor $G:$ pStone $\rightarrow$ BoolRng satisfies (SW).

Let $A$ be a pointed Stone-space and let $M \hookrightarrow G(A)$ be a subring of $G(A)$ such that the source $(h)_{h \in M}$ is point separating. We denote the chosen element of $A$ by $a_{0} .\langle M\rangle$ denotes the unital subring, generated by $M$, of the unital Boolean ring of all continuous maps from $A$ to $\tilde{A}$. We have

$$
\langle M\rangle=M \cup\{1+h \mid h \in M\} .
$$

Let $f: A \rightarrow \tilde{A}$ be a pStone-morphism. From Example 2 we know that $\langle M\rangle$ contains all continuous functions, hence there exists a function $g \in M$ such that

$$
f=g \quad \text { or } \quad f=1+g .
$$

But $f=1+g$ is impossible since we have $0=f\left(a_{0}\right) \neq 1+q\left(a_{0}\right)=1$.

Since $\tilde{A}$ is a regular cogenerator of pStone, $(\tilde{A}, \tilde{B})$ induces a dual equivalence between pStone and BoolRng. 
PROPOSITION 3.6. Assume that the given dual adjunction is a dual equivalence and $G\left(\mathbb{M}_{\mathbf{A}}\right) \subset \mathbb{M}_{\mathbf{B}}$-ExtrEpi. Then $G$ satisfies (SW).

Proof. Let $A$ be an A-object and let $m: M \rightarrow G(A)$ be a $\mathbb{M}_{\mathbf{B}}$-morphism such that the source $(m(f))_{f \in V(M)}$ belongs to $\mathcal{M}_{\mathbf{A}}$. Without loss of generality we may assume that $M=G(B)$ for an A-object $B$ and $m=G(e)$ for a A-morphism $e: A \rightarrow B$. Hence we have

$$
(m(f))_{f \in V(M)}=(f \circ e)_{f \in \operatorname{hom}(B, \tilde{A})} \in \mathcal{M}_{\mathbf{A}}
$$

and therefore $e \in \mathbb{M}_{\mathbf{A}}$. By our assumption we have $m=G(e) \in \mathbb{M}_{\mathbf{B}}$-ExtrEpi and $m$ is an isomorphism.

PROPOSITION 3.7. If $G$ satisfies (SW) then $G\left(\mathcal{M}_{\mathbf{A}}\right) \subset \mathbb{M}_{\mathbf{B}}$-ExtrEpiSink.

Proof. Let $\left(f_{i}: A \rightarrow A_{i}\right)_{i \in I}$ be an $\mathcal{M}_{\mathbf{A}}$-source. We can factorize the (small) sink $\left(G\left(f_{i}\right): G\left(A_{i}\right) \rightarrow G(A)\right)_{i \in I}$ by an $\mathbb{M}_{\mathbf{B}}$-extremal epi-sink $\left(g_{i}: G\left(A_{i}\right) \rightarrow M\right)_{i \in I}$ followed by an $\mathbb{M}_{\mathbf{B}}$-morphism $m: M \rightarrow G(A)$. Hence we have

$$
\{m(h) \mid h \in V(M)\} \supset\left\{k \circ f_{i} \mid i \in I, k \in \operatorname{hom}\left(A_{i}, \tilde{A}\right)\right\},
$$

the source $(m(h))_{h \in V(M)}$ belongs to $\mathcal{M}_{\mathbf{A}}$. Since $G$ satisfies (SW), $m$ is an isomorphism.

In particular we know now that, if we have a dual equivalence, $G$ satisfies (SW) if and only if $F$ satisfies (SW).

EXAMPLE 3.8. By the above proposition, the contravariant functor $G:$ Priest $\rightarrow$ DLat $_{0,1}$ of Example 3.5(3) sends cofiltered limits to collectively surjective sinks. Moreover, since the underlying Stone-space of $\tilde{A}$ is, as a finite space, finitely co-presentable in Stone, $\tilde{A}$ is finitely copresentable in Priest.

For each finite $E \in \mathrm{Ob}$ Priest, there obviously exists an equalizer diagram

$$
E \stackrel{e}{\longrightarrow} \tilde{A}^{n} \stackrel{h}{\stackrel{h}{\longrightarrow}} \tilde{A}^{m}
$$

with $n, m \in \mathbb{N}$. Hence $E$ is finitely copresentable as well.

In the sequel we will use the following weakening of the Stone-Weierstrass Condition.

DEFINITION 3.9. $G$ satisfies the clone-condition provided that the following holds:

(Cl) For each set $X$, every $\mathbb{M}_{\mathbf{B}}$-morphism $m: M \rightarrow G\left(\tilde{A}^{X}\right)$ is an isomorphism provided that the source $(m(f))_{f \in V(M)}$ contains all projections.

Note that this condition is independent of the choice of $\mathbb{M}_{\mathbf{A}}$. If we choose $\mathbf{B}=\{B \in \operatorname{Ob} \operatorname{Alg} \Sigma \mid \operatorname{hom}(B, \tilde{B})$ is a mono-source $\}$ for a given signature $\Sigma$ and 
$\Sigma$-algebra $\tilde{B}$ and $\mathbb{M}_{\mathbf{B}}=\operatorname{Mono}(\mathbf{B})$, then $G$ satisfies the Clone-condition if and only if

$$
V\left(\mathrm{Cl}_{X}(\tilde{B})\right)=U\left(\operatorname{hom}\left(\tilde{A}^{X}, \tilde{A}\right)\right)
$$

for each set $X$. This justifies our choice of the name "clone-condition".

LEMMA 3.10. If the given dual adjunction is a dual equivalence, then $G$ satisfies $(\mathrm{Cl})$.

Proof. Let $X$ be a set and let $m: M \rightarrow G\left(\tilde{A}^{X}\right)$ be an $\mathbb{M}_{\mathbf{B}}$-morphism such that $(m(f))_{f \in V(M)}$ contains all projections. Without loss of generality we may assume that $M=G(A)$ for an $\mathbf{A}$-object $A$ and $m=G(e)$ for an A-epimorphism $e: \tilde{A}^{X} \rightarrow$ $A$. Hence we have

$$
(m(f))_{f \in V(M)}=(f \circ e)_{f \in \operatorname{hom}(B, \tilde{A})} .
$$

$e$ is an extremal monomorphism as well, since $(m(f))_{f \in V(M)}$ contains all projections.

PROPOSITION 3.11. If $G$ satisfies $(\mathrm{Cl})$ and $G\left(\mathbb{M}_{\mathbf{A}}\right) \subset \mathbb{M}_{\mathbf{B}}$-ExtrEpi, then $G$ satisfies (SW).

Proof. Let $A$ be an A-object and let $m: M \rightarrow G(A)$ be an $\mathbb{M}_{\mathbf{A}}$-morphism such that the source $(m(f))_{f \in V(M)}$ belongs to $\mathcal{M}_{\mathbf{A}}$. Hence we have an $\mathbb{M}_{\mathbf{A}}$-morphism $e: A \rightarrow \tilde{A}^{V(M)}$ making the diagram

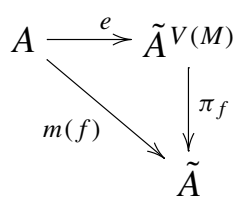

commutative for each $f \in V(M)$. We can form the pullback

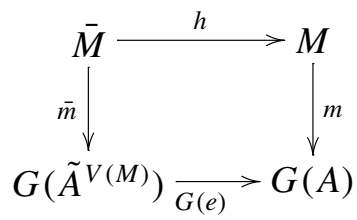

of $G(e): G\left(\tilde{A}^{V(M)}\right) \rightarrow G(A)$ and $m: M \rightarrow G(A)$ in $\mathbf{B} . \mathbb{M}_{\mathbf{B}}$ is stable under pullbacks, hence we have $\bar{m} \in \mathbb{M}_{\mathbf{B}}$. Fullbacks are concrete in $(\mathbf{B}, V)$, therefore

$$
\{\bar{m}(g) \mid g \in V(M)\}=\left\{k: \tilde{A}^{V(M)} \rightarrow \tilde{A} \mid k \circ e \in m[V(M)]\right\} .
$$

The equation above tells us that the set $\{\bar{m}(g) \mid g \in V(\bar{M})\}$ contains all projections, therefore $\bar{m}$ is an isomorphism. By our assumption we have $G(e) \in \mathbb{M}_{\mathbf{B}}$-ExtrEpi, hence also $m \in \mathbb{M}_{\mathbf{B}}$-ExtrEpi. 


\section{Applications}

PROPOSITION 4.1. Let $(\mathbf{C}, W)$ be a concrete category over Set and let $\tilde{C} \in \mathrm{Ob} \mathbf{C}$ such that $(\mathbf{C}, W)$ is concretely $\tilde{C}$-complete and $\tilde{C}$ is a regular injective regular cogenerator of $\mathbf{C}$. Then $(\mathbf{C}, W)$ is concretely complete and the class of regular monomorphisms of $\mathbf{C}$ is closed under composition.

Proof. Since $\tilde{C}$ is a regular cogenerator of $\mathbf{C}$, we can present each $\mathbf{C}$-object $C$ as an equalizer

$$
C \stackrel{e}{\longrightarrow} \tilde{C}^{X} \stackrel{f}{\underset{g}{\longrightarrow}} \tilde{C}^{Y}
$$

of a morphism pair $(f, g)$ between powers of $\tilde{C}$. This enables us to construct products and equalizers in $\mathbf{C}$ through powers of $\tilde{C}$ and equalizers of morphism pairs between powers of $\tilde{C}$ (see [4], Satz 1.12). Moreover, products and equalizers are concrete in $(\mathbf{C}, W)$ since

- powers of $\tilde{C}$ and equalizers of morphism pairs between powers of $\tilde{C}$ are concrete in $(\mathbf{C}, W)$ and

- the underlying limit in Set can be constructed in exactly the same way.

Finally, $\left(\mathbf{C}^{\mathrm{op}}, \operatorname{hom}(, \tilde{C})\right)$ is a quasi-variety and therefore the class of regular epimorphisms of $\mathbf{C}^{\mathrm{op}}$ is closed under composition.

Putting together what we have we get the following theorem.

THEOREM 4.2. Let $(\mathbf{A}, U)$ be a concrete category over Set and let $\tilde{A} \in \mathrm{Ob} \mathbf{A}$. Let $\Sigma$ be a signature, let $\tilde{B}$ be a $\Sigma$-algebra with underlying set $U(\tilde{A})$ and let

$$
\mathbf{B}=\{B \in \operatorname{Ob} \operatorname{Alg} \Sigma \mid \operatorname{hom}(B, \tilde{B}) \text { is a mono-source }\} .
$$

$V: \mathbf{B} \rightarrow$ Set denotes the usual forgetful functor. The following are equivalent.

(1) $(\tilde{A}, \tilde{B})$ induces a natural duality

$$
(\mathbf{A}, U) \stackrel{\eta}{\Rightarrow} \underset{F}{\stackrel{G}{\rightleftarrows}} \stackrel{\varepsilon}{\rightleftarrows}(\mathbf{B}, V) .
$$

(2) The following three conditions are fulfilled.

(a) $(\mathbf{A}, U)$ is concretely $\tilde{A}$-complete.

(b) $\tilde{A}$ is a regular injective regular cogenerator of $\mathbf{A}$.

(c) For each set $X, V\left(\mathrm{Cl}_{X}(\tilde{B})\right)=U\left(\operatorname{hom}\left(\tilde{A}^{X}, \tilde{A}\right)\right)$.

We now consider the case that we already have a duality on the finite level and we wish to extend it to all objects. More precisely, we assume that $G$ and $F$ satisfy the Stone-Weierstrass Condition for all finite objects and we give conditions which imply that $G$ and $F$ satisfy (SW). 
DEFINITION 4.3. $G$ satisfies the Stone-Weierstrass Condition for finite objects provided that the following holds:

$(\mathrm{SW})_{\text {fin }}$ For each A-object $A$ with finite underlying set $U(A)$, every $\mathbb{M}_{\mathbf{B}}$-morphism $m: M \rightarrow G(A)$ is an isomorphism provided that $(m(f))_{f \in V(M)} \in \mathcal{M}_{\mathbf{A}}$.

DEFINITION 4.4. $G$ satisfies the finite clone-condition provided that the following holds:

$(\mathrm{Cl})_{\text {fin }}$ For each finite set $X$, every $\mathbb{M}_{\mathbf{B}}$-morphism $m: M \rightarrow G\left(\tilde{A}^{X}\right)$ is an isomorphism provided that the source $(m(f))_{f \in V(M)}$ contains all projections.

$\tilde{A}$ is called abstractly cofinite if, for each set $X$ and each A-morphism $f: \tilde{A}^{X} \rightarrow$ $\tilde{A}$, there exists a finite subset $S \subset X$ and an A-morphism $f^{\#}: \tilde{A}^{S} \rightarrow \tilde{A}$ such that $f=f^{\#} \circ \pi_{S}$. We have the following fact.

LEMMA 4.5. G satisfies $(\mathrm{Cl})$ provided that it satisfies $(\mathrm{Cl})_{\mathrm{fin}}$ and $\tilde{A}$ is abstractly cofinite.

In addition to our basic situation (Assumption 3.2) we now assume that $U(\tilde{A})=$ $V(\tilde{B})$ is finite, $\mathbb{M}_{\mathbf{A}}=\operatorname{Embed}(\mathbf{A})$ and $(\mathbf{A}, U)$ has (Surj, Embed)-factorizations. Furthermore, the functor $U: \mathbf{A} \rightarrow$ Stone factorizes over the canonical forgetful functor $\left.\right|_{\_} \mid:$Stone $\rightarrow$ Set, i.e., there exists a functor $U^{*}: \mathbf{A} \rightarrow$ Stone such that $U=\left|\_\right| \circ U^{*}$.

Note that, under the given conditions, $\tilde{A}$ is abstractly cofinite. The following characterization of cofiltered limits in $\mathbf{C o m p}_{2}$ (see [2]) will be useful in the sequel.

PROPOSITION 4.6. Let $D: I \rightarrow \mathbf{C o m p}_{2}$ be a cofiltered diagram and let $\mathscr{L}=$ $\left(p_{i}: L \rightarrow D(i)\right)_{i \in I}$ be a compatible cone for $D$. The following are equivalent.

(1) $\mathcal{L}$ is a limit of $D$.

(2) The following two conditions are fulfilled.

(a) $\mathcal{L}$ is a mono-source.

(b) For each $i \in I$, the image of $p_{i}$ is equal to the intersection of the images of all $D(k)$ for all I-morphisms $k: j \rightarrow i$ with codomain $i$, i.e.,

$$
\operatorname{Im} p_{i}=\bigcap_{\substack{k \rightarrow i \\ j \rightarrow i}} \operatorname{Im} D(k) .
$$

Note that this description is dual to the description of filtered colimits in Set. To see this, recall that a compatible cocone $\left(c_{i}: D(i) \rightarrow C\right)_{i \in I}$ for a filtered diagram $D: I \rightarrow$ Set is a colimit of $D$ if and only if the following holds:

(1) $\left(c_{i}: D(i) \rightarrow C\right)_{i \in I}$ is an epi-sink.

(2) For each $i \in I$ and all $x, y \in D(i)$

$$
c_{i}(x)=c_{i}(y) \Leftrightarrow \exists(i \stackrel{k}{\rightarrow} j) \in I D(k)(x)=D(k)(y) .
$$


But the second condition just means that the coimage of $c_{i}$ is equal to the cointersection of the coimages of all $D(k)$ for all $I$-morphisms $k: i \rightarrow j$ with domain $i$.

COROLLARY 4.7. For each $A \in \mathrm{Ob} \mathbf{A}$, the canonical diagram of $A$ with respect to the (essentially small) full subcategory of all finite objects of $(\mathbf{A}, U)$ is cofiltered and $A$ is its canonical limit.

LEMMA 4.8. If each embedding of $(\mathbf{A}, U)$ with finite domain and codomain is a regular monomorphism, then each embedding of $(\mathbf{A}, U)$ is a regular monomorphism.

Proof. Let $m: A^{\prime} \rightarrow A$ be an embedding of (A, $\left.U\right)$. There exists a cofiltered diagram $D: I \rightarrow \mathbf{A}$ and a limit cone $\left(p_{i}: A \rightarrow D(i)\right)_{i \in I}$ with domain $A$ such that, for each $i \in I, U D(i)$ is finite. Let, for each $i \in I$,

$$
A^{\prime} \stackrel{\bar{p}_{i}}{\rightarrow} M_{i} \stackrel{m_{i}}{\rightarrow} D(i)
$$

be a (Surj, Embed)-factorization of $p_{i} \circ m$. It defines a new diagram

$$
D^{*}: I \rightarrow \mathbf{A}, \quad(i \stackrel{k}{\rightarrow} j) \mapsto\left(M_{i} \stackrel{d_{k}}{\rightarrow} M_{j}\right)
$$

and a natural transformation $\left(m_{i}\right)_{i \in I}: D^{*} \rightarrow D$, whereby $d_{k}$ is defined by the (Surj, Embed)-diagonalization property. The cone $\left(\bar{p}_{i}: A^{\prime} \rightarrow D^{*}(i)\right)_{i \in I}$ is by construction compatible for $D^{*}$, point separating and $U$-initial and, for each $i \in$ $I, \bar{p}_{i}$ is surjective. Therefore it is a limit of $D^{*}$. For each $i \in I, m_{i}$ is a regular monomorphism and therefore $m=\lim _{I} m_{i}$ is a regular monomorphism.

LEMMA 4.9. Let $M \in \mathrm{Ob} \mathbf{A}$ such that hom(_, M) sends cofiltered limits with surjective projections to epi-sinks in Set. If $M$ is injective with respect to all embeddings of $(\mathbf{A}, U)$ with finite domain and codomain, then $M$ is injective with respect to all embeddings of (A, $U)$.

Proof. The proof is analogous to the proof of Theorem 2.2.7 of [3].

EXAMPLE 4.10. The two lemmas above allow us to transport some well-known results about Pos to Priest, since both categories (almost) coincide on the finite level. Especially we have in mind:

(1) Each embedding of Priest is a regular monomorphism.

(2) A finite object $A \in \mathrm{Ob}$ Priest is injective w.r.t. embeddings if and only if $A$ is a (complete) lattice.

(3) A finite object $A \in \mathrm{Ob}$ Priest is a regular injective regular cogenerator of Priest if and only if $A$ contains at least two elements and it is a lattice.

PROPOSITION 4.11. Assume that hom(_, $\tilde{A})$ sends cofiltered limits with surjective projections to epi-sinks in Set. $G$ satisfies (SW) if and only if $G$ satisfies $(\mathrm{SW})_{\mathrm{fin}}$. 
Proof. $G$ satisfies $(\mathrm{SW})_{\mathrm{fin}}$, hence it satisfies $(\mathrm{Cl})_{\mathrm{fin}}$ and sends embeddings with finite domain and codomain to $\mathbb{M}_{\mathbf{B}}$-extremal epimorphisms. From Lemma 4.5 we know that $G$ satisfies $(\mathrm{Cl})$. According to Proposition 3.11 we have to show that $G$ sends all embeddings to $\mathbb{M}_{\mathbf{B}}$-extremal epimorphisms.

Let $m: M \rightarrow L$ be an embedding in $(\mathbf{A}, U)$. There exists a cofiltered diagram $D: I \rightarrow \mathbf{A}$ and a limit cone $\left(p_{i}: L \rightarrow D(i)\right)_{i \in I}$ with domain $L$ such that, for each $i \in I, U D(i)$ is finite. Let, for each $i \in I$,

$$
M \stackrel{\bar{p}_{i}}{\rightarrow} M_{i} \stackrel{m_{i}}{\rightarrow} D(i)
$$

be a (Surj, Embed)-factorization of $p_{i} \circ m$. It defines a new diagram

$$
D^{*}: I \rightarrow \mathbf{A}, \quad(i \stackrel{k}{\rightarrow} j) \mapsto\left(M_{i} \stackrel{d_{k}}{\rightarrow} M_{j}\right)
$$

with limit cone $\left(\bar{p}_{i}: M \rightarrow D^{*}(i)\right)_{i \in I}$ and a natural transformation $\left(m_{i}\right)_{i \in I}: D^{*} \rightarrow D$. We apply $G$ to this situation. For each $i \in I$ we get the following commutative diagram.

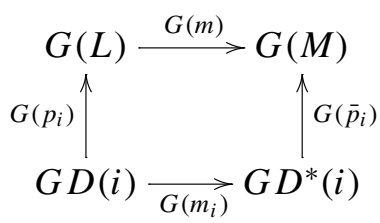

Since the sink $\left(G\left(\bar{p}_{i}\right): G D^{*}(i) \rightarrow G(L)\right)_{i \in I}$ is collectively surjective and $G\left(m_{i}\right)$ is an $\mathbb{M}_{\mathbf{B}}$-extremal epimorphism for each $i \in I$, we conclude that $G(m)$ is an $\mathbb{M}_{\mathbf{B}}$-extremal epimorphism.

PROPOSITION 4.12. Assume that each $B \in \mathrm{Ob} \mathbf{B}$ is a filtered colimit of finite objects. $F$ satisfies $(\mathrm{SW})$ if and only if $F$ satisfies $(\mathrm{SW})_{\mathrm{fin}}$.

Proof. Let $B$ be a $\mathbf{B}$-object. There exist a filtered diagram $D: I \rightarrow \mathbf{B}$ and a colimit cocone $\left(c_{i}: D(i) \rightarrow B\right)_{i \in I}$ such that, for each $i \in I, V D(i)$ is finite. Since $\mathbf{B}$ has the (M-ExtrEpi, $\mathbb{M}$ )-factorization structure we may assume that $c_{i} \in \mathbb{M}_{\mathbf{B}}$ for each $i \in I$.

Let $m: M \rightarrow F(B)$ be an embedding in $(\mathbf{A}, U)$ such that the source

$$
(m(f))_{f \in U(M)}
$$

belongs to $\mathcal{M}_{\mathbf{B}} . F$ sends colimits of $\mathbf{B}$ to limits of $\mathbf{A}$, in particular the cone $\left(F\left(c_{i}\right)\right.$ : $F(B) \rightarrow F D(i))_{i \in I}$ is a limit of $F D$ in $\mathbf{A}$. Let, for each $i \in I$,

$$
M \stackrel{p_{i}}{\rightarrow} M_{i} \stackrel{m_{i}}{\rightarrow} F D(i)
$$

be a (Surj, Embed)-factorization of $F\left(c_{i}\right) \circ m$. As above it defines a new diagram

$$
D^{*}: I \rightarrow \mathbf{A}, \quad(i \stackrel{k}{\rightarrow} j) \mapsto\left(M_{i} \stackrel{d_{k}}{\rightarrow} M_{j}\right)
$$


with limit cone $\left(p_{i}: M \rightarrow D^{*}(i)\right)_{i \in I}$ and a natural transformation $\left(m_{i}\right)_{i \in I}: D^{*} \rightarrow$ $F D$. For each $i \in I$ we have the following commutative diagram.

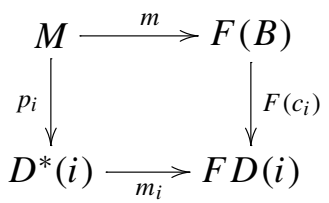

For each $i \in I, m_{i}$ is an embedding and the source $\left(m_{i}(f)\right)_{f \in V\left(M_{i}\right)}$ belongs to $\mathcal{M}_{\mathbf{B}}$ since we have

$$
\left\{m_{i}(f) \mid f \in V\left(M_{i}\right)\right\}=\left\{m(h) \circ c_{i} \mid h \in V(M)\right\} .
$$

But $F$ satisfies $(\mathrm{SW})_{\text {fin }}$ so each $m_{i}(i \in I)$ is an isomorphism. We conclude that $m=\lim _{I} m_{i}$ is an isomorphism.

The following results help to establish the cogenerator property of $\tilde{A}$ resp. $\tilde{B}$.

LEMMA 4.13. Let $(\mathbf{C}, W)$ be a concretely complete category over Set such that each $C \in \mathrm{Ob} \mathbf{C}$ is a filtered colimit of finite objects and $W$ sends filtered colimits to epi-sinks. $\mathbb{M}_{\mathbf{C}}$ denotes a class of $\mathbf{C}$-morphisms satisfying (*) (see beginning of Section 3). Let $M$ be a $\mathbf{C}$-object with finite underlying set $W(M)$. If $M$ is injective with respect to all $\mathbb{M}_{\mathbf{C}}$-morphisms with finite domain and codomain, then $M$ is injective with respect to all $\mathbb{M}_{\mathbf{C}}$-morphisms.

Proof. The proof is analogous to the proof of Lemma 2.2.9 of [3].

PROPOSITION 4.14. Let $(\mathbf{C}, W)$ be a concretely complete category over Set and let $\mathbb{M}_{\mathbf{C}}$ be a class of $\mathbf{C}$-morphisms satisfying (*). Let $\tilde{C}$ be a $\mathbf{C}$-object with finite underlying set $W(\tilde{C})$. Assume that the following four conditions are fulfilled.

(1) $W: \mathbf{C} \rightarrow$ Set sends filtered colimits to epi-sinks.

(2) Each $C \in \mathrm{Ob} \mathbf{C}$ is a filtered colimit of finite objects.

(3) $\tilde{C}$ is injective with respect to all $\mathbb{M}_{\mathbf{C}}$-morphisms with finite domain and codomain.

(4) Let $D: I \rightarrow \mathbf{C}$ be a directed diagram consisting of $\mathbb{M}_{\mathbf{C}}$-morphisms and let $\left(c_{i}: D(i) \rightarrow C\right)_{i \in I}$ be a colimit cocone such that $c_{i} \in \mathbb{M}_{\mathbf{C}}$ for each $i \in I$. For any compatible cocone $\left(f_{i}: D(i) \rightarrow C^{\prime}\right)_{i \in I}$ such that $f_{i} \in \mathbb{M}_{\mathbf{C}}$ for each $i \in I$, the induced morphism $\left[f_{i}\right]_{i \in I}: C \rightarrow C^{\prime}$ belongs to $\mathbb{M}_{\mathbf{C}}$.

Then the source $\operatorname{hom}(C, \tilde{C})$ belongs to $\mathcal{M}_{\mathbf{C}}$ for each $C \in \mathrm{Ob} \mathbf{C}$ provided that the source hom $\left(C_{0}, \tilde{C}\right)$ belongs to $\mathcal{M}_{\mathbf{C}}$ for each finite $C_{0} \in \mathrm{Ob} \mathbf{C}$.

Proof. Let $C$ be a $\mathbf{C}$-object. There exists a directed diagram $D: I \rightarrow \mathbf{C}$ and a colimit cocone $\left(c_{i}: D(i) \rightarrow C\right)_{i \in I}$ with codomain $C$ such that $W D(i)$ is finite for each $i \in I$. We will first show that hom $(C, \tilde{C})$ is point separating. Let $x, y \in W(C)$ such that $x \neq y$. There exist an element $i_{0} \in I$ and elements $x_{0}, y_{0} \in W D\left(i_{0}\right)$ such 
that $c_{i_{0}}\left(x_{0}\right)=x$ and $c_{i_{0}}\left(y_{0}\right)=y$. We may, without loss of generality, assume that $i_{0}$ is the smallest element of $I$. For each $i \in I, k_{i}: i_{0} \rightarrow i$ denotes the unique morphism from $i_{0}$ to $i$. We have $D\left(k_{i}\right)\left(x_{0}\right) \neq D\left(k_{i}\right)\left(y_{0}\right)$. The set

$$
D^{*}(i)=\left\{f \in \operatorname{hom}(D(i), \tilde{C}) \mid f \circ D\left(k_{i}\right)\left(x_{0}\right) \neq f \circ D\left(k_{i}\right)\left(y_{0}\right)\right\}
$$

is non-empty and finite for each $i \in I$. For each $k: i \rightarrow j$ in $I$ and each $f \in D^{*}(j)$ holds

$$
\begin{aligned}
(f \circ D(k)) \circ D\left(k_{i}\right)\left(x_{0}\right) & =f \circ D\left(k_{j}\right)\left(x_{0}\right) \neq f \circ D\left(k_{j}\right)\left(y_{0}\right) \\
& =(f \circ D(k)) \circ D\left(k_{i}\right)\left(y_{0}\right),
\end{aligned}
$$

hence we have $f \circ D(k) \in D^{*}(i)$. Let us define the functor

$$
D^{*}: I^{\text {op }} \rightarrow \text { Set },(i \rightarrow j) \mapsto\left(D^{*}(j) \stackrel{\circ D(k)}{\longrightarrow} D^{*}(i)\right) .
$$

The codirected limit of non-empty and finite sets is non-empty, hence there exists

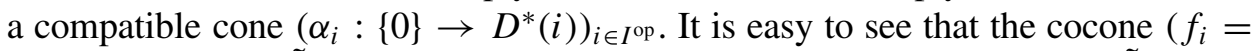
$\left.\alpha_{i}(0): D(i) \rightarrow \tilde{C}\right)_{i \in I}$ is compatible for $D$. Let $f=\left[f_{i}\right]_{i \in I}: C \rightarrow \tilde{C}$ be the induced morphism. We have

$$
f(x)=f \circ c_{i_{0}}\left(x_{0}\right)=f_{i_{0}}\left(x_{0}\right) \neq f_{i_{0}}\left(y_{0}\right)=f \circ c_{i_{0}}\left(y_{0}\right)=f(y) .
$$

We can now assume that $c_{i} \in \mathbb{M}_{\mathbf{C}}$ for each $i \in I$. We have the following commutative diagram.

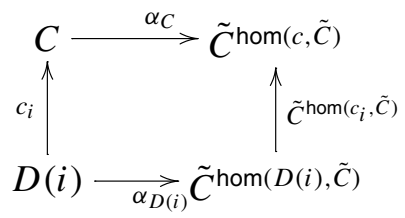

Hereby $\alpha_{C^{\prime}}: C^{\prime} \rightarrow \tilde{C}^{\text {hom }\left(C^{\prime}, \tilde{C}\right)}$ is induced by the source hom $\left(C^{\prime}, \tilde{C}\right)$ for each $C^{\prime} \in \mathrm{Ob} \mathbf{C}$. By the previous lemma, $\tilde{C}$ is $\mathbb{M}_{\mathbf{C}}$-injective and therefore $\operatorname{hom}\left(c_{i}, \tilde{C}\right)$ is surjective, hence we have $\tilde{C}^{\text {hom }\left(c_{i}, \tilde{C}\right)} \in \mathbb{M}_{\mathbf{C}}$. Since $\alpha_{D(i)} \in \mathbb{M}_{\mathbf{C}}$, by assumption, for each $i \in I$, we conclude that $\alpha_{C} \in \mathbb{M}_{\mathbf{C}}$.

PROPOSITION 4.15. Let $\Sigma=\left(\Sigma_{\mathrm{op}}, \sigma\right)$ be a finitary signature and let $\mathrm{g}$ be a set of $\Sigma$-equations such that $\Sigma_{\mathrm{op}}$ is finite and $\sigma(\delta) \leqslant 2$ for each $\delta \in \Sigma_{\mathrm{op}}$. We put

$$
U=\left\{\delta \in \Sigma_{\mathrm{op}} \mid \sigma(\delta)=1\right\} \text { and } B=\left\{\delta \in \Sigma_{\mathrm{op}} \mid \sigma(\delta)=2\right\} .
$$

Assume that the following conditions are satisfied.

(1) For each $\beta \in B$, the associative law

$$
\beta(x, \beta(y, z))=\beta(\beta(x, y), z)
$$

is satisfied. 
(2) There exists a total order $\beta_{1}<\beta_{2}<\cdots<\beta_{n}(n \in \mathbb{N})$ on $B$ such that, for all $1 \leqslant i<j \leqslant n$, the distributive laws

$$
\beta_{j}\left(x, \beta_{i}(y, z)\right)=\beta_{i}\left(\beta_{j}(x, y), \beta_{j}(x, z)\right)
$$

and

$$
\beta_{j}\left(\beta_{i}(x, y), z\right)=\beta_{i}\left(\beta_{j}(x, z), \beta_{j}(y, z)\right)
$$

are satisfied.

(3) $U$ contains the identity and is closed under composition.

(4) The "de Morgan laws" are satisfied, i.e., for each $\alpha \in U$ and each $\beta \in B$, there exist operation symbols $\beta^{\prime} \in B$ and $\alpha^{\prime}, \alpha^{\prime \prime} \in U$ such that

$$
\alpha(\beta(x, y))=\beta^{\prime}\left(\alpha^{\prime}(x), \alpha^{\prime \prime}(y)\right)
$$

or

$$
\alpha(\beta(x, y))=\beta^{\prime}\left(\alpha^{\prime}(y), \alpha^{\prime \prime}(x)\right) .
$$

Then each $A \in \mathrm{Ob} \operatorname{StoneA} \operatorname{Ig}(\Sigma, \mathcal{G})$ is a cofiltered limit of finite algebras.

Proof. See Theorem VI 2.9 of [7].

As an application of these results we will now show that each strong duality of [3] gives rise to a new duality by "structure interchange". Let us first recall very briefly some basic facts of [3].

Let $G, H$ and $R$ be disjoint sets of respectively finitary operation symbols, of finitary partial operation symbols and of finitary relation symbols and let $\alpha: G \cup$ $H \cup R \rightarrow \mathbb{N}$ be a function which assigns to each symbol an arity. A structured Stone-space of type $(G, H, R)$ is a structure

$$
A=\left(|A|, G^{A}, H^{A}, R^{A}, \tau^{A}\right)
$$

consisting of a Stone-space $\left(|A|, \tau_{A}\right)$ and

(1) a family $G^{A}=\left(g^{A}\right)_{g \in G}$ of continuous operations $g^{A}:|A|^{\alpha(g)} \rightarrow|A|$,

(2) a family $H^{A}=\left(h^{A}\right)_{h \in H}$ of continuous partial operations $h^{A}: \operatorname{dom}\left(h^{A}\right) \rightarrow|A|$ on $|A|$ with closed domain $\operatorname{dom}\left(h^{A}\right) \subset|A|^{\alpha(h)}$ and

(3) a family $R^{A}=\left(r^{A}\right)_{r \in R}$ of closed relations $r^{A} \subset|A|^{\alpha(r)}$.

Let $A=\left(|A|, G^{A}, H^{A}, R^{A}, \tau^{A}\right)$ and $A^{\prime}=\left(\left|A^{\prime}\right|, G^{A^{\prime}}, H^{A^{\prime}}, R^{A^{\prime}}, \tau^{A^{\prime}}\right)$ be structured Stone-spaces of the same type $(G, H, R)$. A map $f:|A| \rightarrow\left|A^{\prime}\right|$ is a morphism $f: A \rightarrow A^{\prime}$ if $f$ is continuous and preserves all operations, partial operations and relations. For a given type $(G, H, R)$ we can form the category $\operatorname{StoneStr}(G, H, R)$ with objects all Stone-spaces of type $(G, H, R)$ and morphisms all morphisms between these structures $\mathbb{M}_{0}$ denotes the class of all injective morphisms

$$
f:\left(|A|, G^{A}, H^{A}, R^{A}, \tau^{A}\right) \rightarrow\left(\left|A^{\prime}\right|, G^{A^{\prime}}, H^{A^{\prime}}, R^{A^{\prime}}, \tau^{A^{\prime}}\right)
$$


such that the structure of $A$ is exactly the restriction of the structure of $A^{\prime}$. Obviously, $\mathbb{M}_{0}$ satisfies the conditions $(*)$. Assume that a type $(G, H, R)$ is given and let $\tilde{M}=\left(M, G^{\tilde{M}}, H^{\tilde{M}}, R^{\tilde{M}}, \tau^{\tilde{M}}\right)$ be a structured Stone-space of type $(G, H, R)$. $\mathcal{X}$ denotes the full subcategory of $\operatorname{StoneStr}(G, H, R)$ with objects all structured Stone-spaces $A$ of type $(G, H, R)$ such that $\operatorname{hom}(A, \tilde{M}) \in \mathcal{M}_{0}$, i.e., all $\mathbb{M}_{0}$-subobjects of some power of $\tilde{M}$. The aim is now to find, for a given finitary signature $\Sigma$ and a given finite $\Sigma$-algebra $\bar{M}=\left(M, \delta^{\bar{M}}\right)$, a type $(G, H, R)$ and a structured Stone-space $\tilde{M}=\left(M, G^{\tilde{M}}, H^{\tilde{M}}, R^{\tilde{M}}, \tau^{\tilde{M}}\right)$ with carrier $M$ such that $(\tilde{M}, \bar{M})$ induces a duality

$$
\mathcal{X} \stackrel{\varepsilon}{\Rightarrow} \underset{D}{\stackrel{E}{\rightleftarrows}} \stackrel{e}{\rightleftharpoons} \mathcal{A} .
$$

Hereby $\mathcal{A}$ denotes the full subcategory of $\operatorname{Alg} \Sigma$ cogenerated by $\bar{M}$, i.e.,

$$
\mathcal{A}=\{B \in \mathrm{Ob} \operatorname{Alg} \Sigma \mid \operatorname{hom}(B, \bar{M}) \text { is a mono-source }\} .
$$

This duality is called strong if $\tilde{M}$ is in addition injective with respect to $\mathbb{M}_{0}$. Note that such a duality is strong if and only if $E$ and $D$ satisfy (SW) with respect to $\mathbb{M}_{X}=\mathbb{M}_{0} \cap \operatorname{Mor} \mathcal{X}$ and $\mathbb{M}_{\mathcal{A}}=\operatorname{Mono}(\mathcal{A})$.

Assume now that a finitary signature $\Sigma$, a type $(G, H, R)$, a $\Sigma$-algebra $\bar{M}=$ $\left(M, \delta^{\bar{M}}\right)$ and a structured Stone-space $\tilde{M}=\left(M, G^{\tilde{M}}, H^{\tilde{M}}, R^{\tilde{M}}, \tau^{\tilde{M}}\right)$ with a finite set $M$ are given such that $(\tilde{M}, \bar{M})$ induces a strong duality

$$
\mathcal{X} \stackrel{\varepsilon}{\Rightarrow} \underset{D}{\stackrel{E}{\rightleftarrows}} \stackrel{e}{\rightleftharpoons} \mathcal{A} .
$$

We now define the category $\operatorname{Str}(G, H, R)$ of $(G, H, R)$-structured sets in the same way as $\operatorname{StoneStr}(G, H, R)$, just without topology. $\operatorname{Str}(G, H, R)$ is locally finitely presentable and hence complete and cocomplete. It has a canonical forgetful functor to Set which has a left adjoint and preserves filtered colimits. $\mathbb{M}_{1}$ is the class of all $\operatorname{Str}(G, H, R)$-morphisms

$$
f: A=\left(|A|, G^{A}, H^{A}, R^{A}\right) \rightarrow A^{\prime}=\left(\left|A^{\prime}\right|, G^{A^{\prime}}, H^{A^{\prime}}, R^{A^{\prime}}\right)
$$

such that $f$ is injective and the structure of $A$ is exactly the restriction of the structure of $A^{\prime}$.

LEMMA 4.16. Let $D: I \rightarrow \operatorname{Str}(G, H, R)$ be a directed diagram such that $D(k) \in \mathbb{M}_{1}$ for each $k: i \rightarrow j$ from I. Let $\left(c_{i}: D(i) \rightarrow B\right)_{i \in I}$ be a colimit of $D$. Then we have $c_{i} \in \mathbb{M}_{1}$ for each $i \in I$. Let $\left(f_{i}: D(i) \rightarrow B^{\prime}\right)_{i \in I}$ be a compatible cocone for $D$ consisting of $\mathbb{M}_{1}$-morphisms. Then $\left[f_{i}\right]_{i \in I} \in \mathbb{M}_{1}$.

We put $\tilde{A}=\left(M, \delta^{\bar{M}}, \mathcal{P}(M)\right) \in \mathrm{Ob} \operatorname{StoneA} \lg (\Sigma), \tilde{B}=\left(M, G^{\tilde{M}}, H^{\tilde{M}}, R^{\tilde{M}}\right) \in$ $\mathrm{Ob} \operatorname{Str}(G, H, R)$ and define the categories

$$
\begin{aligned}
& \mathbf{A}=\{A \in \mathrm{Ob} \operatorname{StoneAIg}(\Sigma) \mid \operatorname{hom}(A, \tilde{A}) \text { is a mono-source }\}, \\
& \mathbf{B}=\left\{B \in \mathrm{Ob} \operatorname{Str}(G, H, R) \mid \operatorname{hom}(B, \tilde{B}) \in \mathcal{M}_{1}\right\} .
\end{aligned}
$$


$U: \mathbf{A} \rightarrow$ Set and $V: \mathbf{B} \rightarrow$ Set denote the canonical forgetful functors. We put $\mathbb{M}_{\mathbf{A}}=\operatorname{Embed}(\mathbf{A})=\operatorname{Mono}(\mathbf{A})$ and $\mathbb{M}_{\mathbf{B}}=\operatorname{Mor}(\mathbf{B}) \cap \mathbb{M}_{1}$. By construction, $\tilde{A}$ is an initial cogenerator of $(\mathbf{A}, U)$ and $\tilde{B}$ is an $\mathbb{M}_{\mathbf{B}}$-cogenerator of $(\mathbf{B}, V)$. Obviously, each $B \in \mathrm{Ob} \mathbf{B}$ is the directed colimit of all its finite $\mathbb{M}_{\mathbf{B}}$-subobjects. Each surjection in $(\mathbf{A}, U)$ is final and the underlying Stone-space of $\tilde{A}$ is copresentable in Stone, hence the contravariant hom-functor hom $\left(\_, \tilde{A}\right)$ sends cofiltered limits with surjective projections to epi-sinks.

THEOREM 4.17. $(\tilde{A}, \tilde{B})$ induces a natural dual adjunction

$$
(\mathbf{A}, U) \stackrel{\eta}{\Rightarrow} \underset{F}{\stackrel{G}{\rightleftarrows}} \stackrel{\varepsilon}{\rightleftarrows}(\mathbf{B}, V) .
$$

Moreover, for each $A \in \mathrm{Ob} \mathbf{A}$, the source $\left(\mathrm{ev}_{A, a}: G(A) \rightarrow \tilde{B}\right)_{a \in U(A)}$ belongs to $\mathcal{M}_{\mathbf{B}}$ and the functors $G$ and $F$ satisfy (SW).

Proof. For all finite objects $A \in \mathrm{Ob} \mathbf{A}$ and $B \in \mathrm{Ob} \mathbf{B}$, the $V$-structured source $\left(\operatorname{ev}_{A, a}: \operatorname{hom}(A, \tilde{A}) \rightarrow V(\tilde{B})\right)_{a \in U(A)}$ admits an $\mathcal{M}_{1}$-lifting and the $U$-structured source $\left(\operatorname{ev}_{B, b}: \operatorname{hom}(B, \tilde{B}) \rightarrow U(\tilde{A})\right)_{b \in V(B)}$ admits a $U$-initial lifting. Moreover, each $\mathbf{A}$-object $A$ is a codirected limit of finite objects in $(\mathbf{A}, U)$ such that all limit projections are surjective, each $\mathbf{B}$-object $B$ is a concrete directed colimit of finite objects in $(\mathbf{B}, V)$. From that we can prove that the conditions (Init $\mathbf{A}$ ) and (Init $\mathbf{B}$ ) are fulfilled as we are now going to show.

For each $A \in \mathrm{Ob} \mathbf{A}, U_{A}: \operatorname{hom}(A, \tilde{A}) \rightarrow U(\tilde{A})^{U(A)}=V(\tilde{B})^{U(A)}$ denotes the restriction of $U$ and $V_{B}: \operatorname{hom}(B, \tilde{B}) \rightarrow V(\tilde{B})^{V(B)}=U(\tilde{A})^{V(B)}$ denotes, for each $B \in \mathrm{Ob} \mathbf{B}$, the restriction of $V$.

Let $A$ be an A-object, let $D: I \rightarrow \mathbf{A}$ be a codirected diagram and let $\left(p_{i}: A \rightarrow\right.$ $D(i))_{i \in I}$ be a limit of $D$ with domain $A$ such that, for each $i \in I, p_{i}$ is surjective. Hence the cocone

$$
\operatorname{hom}\left(p_{i}, \tilde{A}\right): \operatorname{hom}(D(i) \tilde{A}) \rightarrow \operatorname{hom}(A, \tilde{A})
$$

is a colimit of $\operatorname{hom}\left(D\left(\_\right), \tilde{A}\right): I^{\mathrm{op}} \rightarrow$ Set. For each $i \in I$ we have the following commutative diagram.

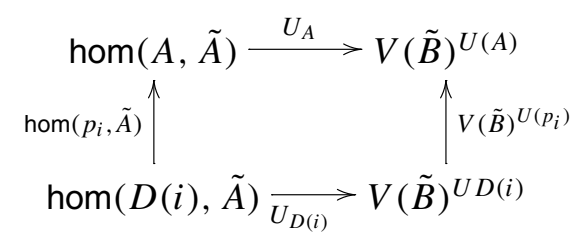

We put $G(A)=$ colim $_{I^{\text {op }}} G D$, whereby the colimit is taken in $\operatorname{Str}(G, H, R)$. We have $V G(A)=\operatorname{hom}(A, \tilde{A})$. For each $i \in I, U_{D(i)}$ is an $\mathbb{M}_{\mathbf{B}}$-morphism $U_{D(i)}$ : $G D(i) \rightarrow \tilde{B}^{U D(i)}$ and $V(\tilde{B})^{U\left(p_{i}\right)}$ underlies the $\mathbb{M}_{\mathbf{B}}$-morphism $\tilde{B}^{U\left(p_{i}\right)}: \tilde{B}^{U D(i)} \rightarrow$ $\tilde{B}^{U(A)}$. Hence $U_{A}: G(A) \rightarrow \tilde{B}^{U(A)}$ is an $\mathbb{M}_{\mathbf{B}}$-morphism as well, in particular $G(A) \in \mathrm{Ob} \mathbf{B}$. 
The same idea is essentially the key to prove that (Init $\mathbf{B}$ ) is fulfilled. Let $B$ be a $\mathbf{B}$-object, let $D: I \rightarrow \mathbf{B}$ be a directed diagram and let $\left(c_{i}: D(i) \rightarrow B\right)_{i \in I}$ be a concrete colimit of $D$ with codomain $B$. For each $i \in I$, the following diagram commutes.

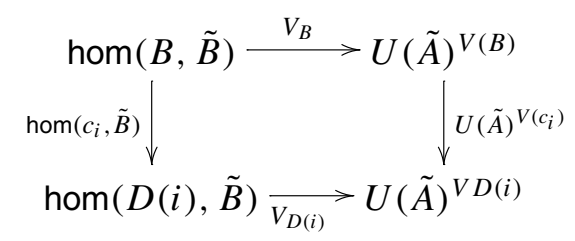

The left-hand and the right-hand side are limit cones since $\left(c_{i}: D(i) \rightarrow B\right)_{i \in I}$ as well as $\left(c_{i}: V D(i) \rightarrow V(B)\right)_{i \in I}$ are colimit cones and hom(_, $\left.\tilde{B}\right)$ and $\tilde{B}$-send colimits to limits. We put $F(B)=\lim _{I^{\text {op }}} F D(i)$. For each $i \in I, V_{D(i)}$ is an Amonomorphism $V_{D(i)}: F D(i) \rightarrow \tilde{A}^{V D(i)}$. We conclude that $V_{B}: F(B) \rightarrow \tilde{A}^{V(B)}$ is an A-monomorphism as well.

According to Propositions 4.11 and 4.12, $G$ and $F$ satisfy (SW).

EXAMPLE 4.18. Let us consider Example 4.3.15 of [3].

A de Morgan algebra $B=(|B|, \vee, \wedge, \neg, 0,1)$ is a bounded distributive lattice with a negation operator " $\neg$ " satisfying

$$
\neg \neg y=y, \neg 0=1, \neg\left(y^{\prime} \vee y\right)=\neg y^{\prime} \wedge \neg y, \neg\left(y^{\prime} \wedge y\right)=\neg y^{\prime} \vee \neg y
$$

for each $y, y^{\prime} \in|B|$. deMorgan denotes the variety of de Morgan algebras. Let $\bar{M}=(\{0, a, b, 1\}, \vee, \wedge, \neg, 0,1)$, where the lattice structure is shown in the diagram

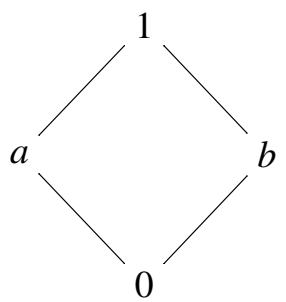

and " $\neg$ " interchanges 0 and 1 and fixes $a$ and $b . \bar{M}$ is a cogenerator of deMorgan.

The objects of $X$ are precisely the tuples $(X, \leqslant, f, \tau)$ consisting of a Priestley space $(X, \leqslant, \tau)$ and an order-reversing homeomorphism $f: X \rightarrow X$ with $f \circ f=$ $\operatorname{id}_{X}$. Let $\tilde{M}=\left(\{0, a, b, 1\}, \leqslant, f_{0}, \tau\right)$, whereby the order relation is shown in the diagram

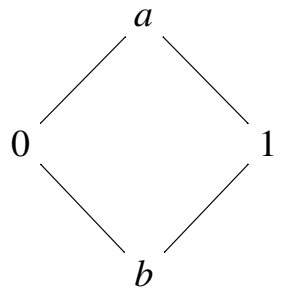


and $f_{0}$ fixes 0 and 1 and interchanges $a$ and $b .(\tilde{M}, \bar{M})$ induces a strong duality ([3], Theorem 4.3.16)

$$
X \stackrel{\varepsilon}{\Rightarrow} \underset{D}{\stackrel{E}{\rightleftarrows}} \stackrel{e}{\models} \mathcal{A} .
$$

From Proposition 4.15 we know that

$$
\tilde{A}=(\{0, a, b, 1\}, \vee, \wedge, \neg, 0,1, \mathcal{P}(\{0, a, b, 1\}))
$$

is a cogenerator of StonedeMorgan, hence we have $\mathbf{A}=$ StonedeMorgan. On the other side we put $\tilde{B}=\left(\{0, a, b, 1\}, \leqslant, f_{0}\right)$, $\mathbf{B}$ is the category of tuples $(Y, \leqslant, f)$ consisting of a set $Y$, an order relation " $\leqslant$ " on $Y$ and an order-reversing bijection $f: Y \rightarrow Y$ with $f \circ f=\operatorname{id}_{Y}$ (Proposition 4.14). By the previous theorem, $(\tilde{A}, \tilde{B})$ induces a natural dual adjunction

$$
(\mathbf{A}, U) \stackrel{\eta}{\Rightarrow} \underset{F}{\stackrel{G}{\rightleftarrows}} \stackrel{\varepsilon}{\rightleftarrows}(\mathbf{B}, V)
$$

and $G$ and $F$ satisfy (SW). We have proved that StonedeMorgan is dually equivalent to $\mathbf{B}$.

\section{Acknowledgements}

The work presented here is part of the author's Ph.D. Thesis [6]. I am grateful to my supervisor, Prof. Dr. Hans-Eberhard Porst, for many fruitful discussions and suggestions.

\section{References}

1. Adámek, J., Herrlich, H. and Strecker, G. E.: Abstract and Concrete Categories, Wiley Interscience, New York, 1990.

2. Bourbaki, N.: Topologie générale (Éléments de Mathématique, Livre III), chapitres 1-2, 3rd edn, Actualités Sci. Ind. 1142, Hermann, Paris, 1961.

3. Clark, D. M. and Davey, B. A.: Natural Dualities for the Working Algebraist, Cambridge University Press, Cambridge, 1997.

4. Gabriel, P. and Ulmer, F.: Lokal präsentierbare Kategorien, Lecture Notes in Math. 221, Springer, New York, 1971.

5. Gelfand, I. M., Rajkov, D. A. and Shilov, G. E.: Commutative normed rings, Uspekhi Mat. Nauk 1(2) (1946), 48-146.

6. Hofmann, D.: Natürliche Dualitäten und das verallgemeinerte Stone-Weierstraß-Theorem, Ph.D. Thesis, University of Bremen, 1999.

7. Johnstone, P. T.: Stone Spaces, Cambridge University Press, Cambridge, 1982.

8. Priestley, H. A.: Representation of distributive lattices by means of ordered Stone spaces, Bull. London Math. Soc. 2 (1970), 186-190.

9. Priestley, H. A.: Ordered topological spaces and the representation of distributive lattices, Proc. London Math Soc. (3) 24 (1972), 507-530. 
10. Stone, M. H.: The theory of representations of Boolean algebras, Trans. Amer. Math. Soc. 40 (1936), 37-111.

11. Stone, M. H.: Applications of the theory of Boolean rings to general topology, Trans. Amer. Math. Soc. 41 (1937), 375-481.

12. Stone, M. H.: The generalized Weierstrass approximation theorem, Math. Mag. 21 (1948), 167-184.

13. Porst, H.-E. and Tholen, W.: Concrete dualities, in Category Theory at Work, Heldermann Verlag, Berlin, 1991, pp. 111-136.

14. Stralka, A.: A partial ordered space which is not a Priestley space, Semigroup Forum 20 (1980), 293-297.

15. Weierstrass, K.: Über die analytische Darstellbarkeit sogenannter willkürlicher Functionen reeler Argumente, Sitzungsber. Kgl. Preuss. Akad. Wiss., Berlin, 1885. 